\title{
ON THE DETERMINANTS OF CERTAIN TOEPLITZ MATRICES
}

BY LARS C. MEJLBO AND PALLE F. SCHMIDT

Communicated by Edwin Moise, October 20, 1960

With a function of the form

$$
\phi(\theta)+g(x)=\sum_{\nu=-\infty}^{\infty} a_{\nu} e^{i \nu \theta}+g(x), \quad 0 \leqq x \leqq 1,
$$

we associate for each $n=0,1,2, \cdots$, a Toeplitz matrix

$$
T_{n}(\phi(\theta)+g(x))=\left\{a_{i-j}+\delta_{i j} g\left(\frac{i}{n+1}\right)\right\}, \quad i, j=0,1, \cdots, n,
$$

where $\delta_{i j}=1$ if $i=j, \delta_{i j}=0$ if $i \neq j$.

Furthermore we define

$$
\begin{aligned}
D_{n}(\phi(\theta)+g(x)) & =\operatorname{det} T_{n}(\phi(\theta)+g(x)), \\
G(\phi(\theta)+g(x)) & =\exp \left\{\frac{1}{2 \pi} \int_{-\pi}^{\pi} \int_{0}^{1} \log (\phi(\theta)+g(x)) d x d \theta\right\}, \\
L(\phi(\theta)+g(x)) & =\lim _{n \rightarrow \infty} \frac{D_{n}(\phi(\theta)+g(x))}{[G(\phi(\theta)+g(x))]^{n+1}},
\end{aligned}
$$

whenever these definitions make sense.

We shall prove that under the conditions

(i) $g(x)$ is real and differentiable for $0 \leqq x \leqq 1$ with $g^{\prime}(x)$ satisfying the Lipschitz condition

$$
\left|g^{\prime}\left(x_{1}\right)-g^{\prime}\left(x_{2}\right)\right|<K\left|x_{1}-x_{2}\right|^{\alpha}, \quad K>0, \alpha>0,
$$

(ii) $\phi(\theta)$ is a trigonometric polynomial of the type

(iii)

$$
\begin{aligned}
\phi(\theta) & =\sum_{\nu=-k}^{k} a_{\nu} e^{i \nu \theta}, & \\
a_{0} & =0, a_{\nu}=a_{-v}, a_{\nu} \text { real, } & \nu=1,2, \cdots, k, \\
& \sum_{\nu=-k}^{k}\left|a_{\nu}\right|<g(x), & \text { for } 0 \leqq x \leqq 1,
\end{aligned}
$$

the limit $L(\phi(\theta)+g(x))$ exists and has the value

$$
\begin{aligned}
& L(\phi(\theta)+g(x)) \\
& \quad=\left(\frac{G(\phi(\theta)+g(0))}{G(\phi(\theta)+g(1))} L(\phi(\theta)+g(0)) L(\phi(\theta)+g(1))\right)^{1 / 2} .
\end{aligned}
$$


(For the existence and the value of $L(\phi(\theta)+g(0))$ and $L(\phi(\theta)+g(1))$, see $[1$, Theorem 5.5, p. 76] or [2].)

Without loss of generality we may assume that

$$
\sum_{\nu=-k}^{k}\left|a_{\nu}\right|+g(x)<1, \quad 0 \leqq x \leqq 1 .
$$

Let

$$
|\phi|(\theta)=\sum_{\nu=-k}^{k}\left|a_{\nu}\right| e^{i v \theta}
$$

We then have

$$
\begin{aligned}
& 0<-\phi(\theta)+1-g(x)<\beta<1, \\
& 0<|\phi|(\theta)+1-g(x)<\beta<1,
\end{aligned}
$$

for $-\pi \leqq \theta \leqq \pi, 0 \leqq x \leqq 1$.

Let us introduce

$$
H_{n}(\phi(\theta)+g(x))=\exp \left\{\frac{1}{2 \pi} \int_{-\pi}^{\pi} \sum_{\mu=0}^{n} \log \left(\phi(\theta)+g\left(\frac{\mu}{n+1}\right)\right) d \theta\right\} .
$$

By use of (i) it is easy to prove that

$$
\lim _{n \rightarrow \infty} \frac{H_{n}(\phi(\theta)+g(x))}{[G(\phi(\theta)+g(x))]^{n+1}}=\left(\frac{G(\phi(\theta)+g(0))}{G(\phi(\theta)+g(1))}\right)^{1 / 2} .
$$

Now we have

$$
\begin{aligned}
\log D_{n}(\phi(\theta)+g(x)) & =-\sum_{p=1}^{\infty} \frac{1}{p} \operatorname{Tr}\left\{T_{n}^{p}(-\phi(\theta)+1-g(x))\right\} \\
\log H_{n}(\phi(\theta)+g(x)) & \\
= & -\sum_{p=1}^{\infty} \frac{1}{p} \sum_{\mu=0}^{n} \frac{1}{2 \pi} \int_{-\pi}^{\pi}\left(-\phi(\theta)+1-g\left(\frac{\mu}{n+1}\right)\right)^{p} d \theta
\end{aligned}
$$

and hence

$$
\log \frac{D_{n}(\phi(\theta)+g(x))}{H_{n}(\phi(\theta)+g(x))}=\sum_{p=1}^{\infty} \frac{1}{p} \sum_{\mu=0}^{n} E_{\mu}(n, p),
$$

where

$$
\begin{aligned}
E_{\mu}(n, p)= & \frac{1}{2 \pi} \int_{-\pi}^{\pi}\left(-\phi(\theta)+1-g\left(\frac{\mu}{n+1}\right)\right)^{p} d \theta \\
& -\left\{T_{n}^{p}(-\phi(\theta)+1-g(x))\right\}_{\mu \mu} .
\end{aligned}
$$

Put $\gamma=\max _{0 \leq x \leq 1}(1-g(x))$. Then 


$$
\left|E_{\mu}(n, p)\right| \leqq \beta^{p}+\frac{1}{2 \pi} \int_{-\pi}^{\pi}(|\phi|(\theta)+\gamma)^{p} d \theta \leqq 2 \beta^{p},
$$

for $n=0,1,2, \cdots ; p=1,2, \cdots ; \mu=0,1,2, \cdots, n$. For $n \geqq 2 p k$ and $p k \leqq \mu \leqq n-p k$ we have

$$
\begin{gathered}
E_{\mu}(n, p)=\sum_{\alpha_{1}+\cdots+\alpha_{p} \infty 0} \prod_{\alpha_{j \neq 0}}\left(-a_{\alpha_{j}}\right) \\
\cdot\left[\prod_{\alpha_{j}=0}\left(1-g\left(\frac{\mu}{n+1}\right)\right)-\prod_{\alpha_{j}=0}\left(1-g\left(\frac{\mu+\alpha_{1}+\cdots+\alpha_{j}}{n+1}\right)\right)\right]
\end{gathered}
$$

from which we get

$$
\left|E_{\mu}(n, p)\right| \leqq \operatorname{constant} \frac{p^{2} k}{n+1} \sum_{\alpha_{1}+\cdots+\alpha_{p}=0} \prod_{\alpha_{j} \neq 0}\left|a_{\alpha_{j}}\right| \prod_{\alpha_{j}=0} \gamma,
$$

where the constant only depends on $g(x)$ and $g^{\prime}(x)$. Hence

$$
\begin{aligned}
\left|E_{\mu}(n, p)\right| & \leqq \text { constant } \frac{p^{2} k}{n+1} \frac{1}{2 \pi} \int_{-\pi}^{\pi}(|\phi|(\theta)+\gamma)^{p} d \theta \\
& \leqq \text { constant } \frac{p^{2} k}{n+1} \beta^{p},
\end{aligned}
$$

for $n \geqq 2 p k$ and $p k \leqq \mu \leqq n-p k$. By combining (4) and (5) we see that (3) is dominated by a convergent series of the form

$$
\text { constant } \sum_{p=1}^{\infty} p \beta^{p} \text {. }
$$

To get a better estimate of $E_{\mu}(n, p)$ for $n \geqq 2 p k$ and $p k \leqq \mu \leqq n-p k$ we let $\sum^{*}$ denote summation over all $\left(\alpha_{1}, \alpha_{2}, \cdots, \alpha_{p}\right) \neq(0,0, \cdots, 0)$ satisfying

$$
\begin{aligned}
& \alpha_{j}=0, \pm 1, \pm 2, \cdots, \pm k, \quad j=1,2, \cdots, p, \\
& \alpha_{1}+\alpha_{2}+\cdots+\alpha_{p}=0,
\end{aligned}
$$

first $\alpha_{j} \neq 0$ is positive.

Then

$$
\begin{aligned}
E_{\mu}(n, p)=\sum^{*} \prod_{\alpha_{j \neq 0}}\left(-a_{\alpha_{j}}\right)[ & 2 \prod_{\alpha_{j}=0}\left(1-g\left(\frac{\mu}{n+1}\right)\right) \\
& -\prod_{\alpha_{j}=0}\left(1-g\left(\frac{\mu+\alpha_{1}+\cdots+\alpha_{j}}{n+1}\right)\right) \\
& \left.-\prod_{\alpha_{j}=0}\left(1-g\left(\frac{\mu-\alpha_{1}-\cdots-\alpha_{j}}{n+1}\right)\right)\right] .
\end{aligned}
$$


From (i) it follows that

$$
g(x+\Delta x)=g(x)+g^{\prime}(x) \Delta x+R(x, \Delta x) \Delta x^{1+\alpha},
$$

where $R(x, \Delta x)$ is bounded. Using this we get

$$
\left|E_{\mu}(n, p)\right| \leqq \text { constant }\left(\frac{1}{n+1}\right)^{1+\alpha} \text {, }
$$

where the constant does not depend on $n$. Hence

$$
\lim _{n \rightarrow \infty} \sum_{\mu=p k}^{n-p k} E_{\mu}(n, p)=0
$$

and therefore

$$
\lim _{n \rightarrow \infty} \sum_{\mu=0}^{n} E_{\mu}(n, p)=\sum_{\mu=0}^{p k-1} u(\mu, p)+\sum_{\mu=0}^{p k-1} v(\mu, p),
$$

where

$$
\begin{aligned}
& u(\mu, p)=\frac{1}{2 \pi} \int_{-\pi}^{\pi}(-\phi(\theta)+1-g(0))^{p} d \theta-\left\{T_{2 p k}^{p}(-\phi(\theta)+1-g(0))\right\}_{\mu \mu}, \\
& v(\mu, p)=\frac{1}{2 \pi} \int_{-\pi}^{\pi}(-\phi(\theta)+1-g(1))^{p} d \theta-\left\{T_{2 p k}^{p}(-\phi(\theta)+1-g(1))\right\}_{\mu \mu} .
\end{aligned}
$$

From (6) we conclude that

$$
\begin{aligned}
\lim _{n \rightarrow \infty} \sum_{p=1}^{\infty} \frac{1}{p} \sum_{\mu=0}^{n} E_{\mu}(n, p) & \\
& =\sum_{p=1}^{\infty} \frac{1}{p} \sum_{\mu=0}^{p k-1} u(\mu, p)+\sum_{p=1}^{\infty} \frac{1}{p} \sum_{\mu=0}^{p k-1} v(\mu, p) \\
& =\frac{1}{2} \log L(\phi(\theta)+g(0))+\frac{1}{2} \log L(\phi(\theta)+g(1))
\end{aligned}
$$

(the last equality being the result of a straightforward computation).

The formula (1) now follows by combining (2), (3), and (7).

\section{REFERENCES}

1. U. Grenander and G. Szegö, Toeplitz forms and their applications, University of California Press, 1958.

2. G. Szegö, On certain hermitian forms associated with the Fourier series of a positive function, Festskrift Marcel Riesz, Lund, 1952.

University of Aarhus, DenMark 